Relations industrielles

Industrial Relations

\title{
Les apprentis scolarisés, par Robert Schiélé et André Moujaret, dans la Collection « L'Enquête et l'Action ", les Éditions ouvrières, Paris, 328 pages.
}

Volume 20, numéro 4, 1965

URI : https://id.erudit.org/iderudit/027640ar

DOI : https://doi.org/10.7202/027640ar

Aller au sommaire du numéro

Éditeur(s)

Département des relations industrielles de l'Université Laval

ISSN

0034-379X (imprimé)

1703-8138 (numérique)

Découvrir la revue

Citer ce compte rendu

(1965). Compte rendu de [Les apprentis scolarisés, par Robert Schiélé et André Moujaret, dans la Collection "L'Enquête et l'Action ", les Éditions ouvrières, Paris, 328 pages.] Relations industrielles / Industrial Relations, 20(4), 724-725. https://doi.org/10.7202/027640ar

Tous droits réservés @ C Département des relations industrielles de l'Université Laval, 1965
Ce document est protégé par la loi sur le droit d'auteur. L'utilisation des services d'Érudit (y compris la reproduction) est assujettie à sa politique d'utilisation que vous pouvez consulter en ligne.

https://apropos.erudit.org/fr/usagers/politique-dutilisation/ 
De foçon plus particulière encore l'auteur se propose de faire connaitre certaines relations empiriquement utilisables pour I'analyse de la substitution entre le capital et le travail, de constater, quantitativement, les différences dans le coût du travail et du capital pour un nombre suffisamment considérable d'industries manufacturières et de dériver des estimations numériques d'élasticité de substitution entre ces deux facteurs, et enfin de montrer la signification de ces découvertes empiriques pour les problèmes de commerce international et de développement économique.

Après un premier chapitre dans lequel Minhas expose les données du problème et l'importance de ce dernier, un second nous fournit un cadre théorique pour l'étude des effets des coûts variables d'un focteur sur le modèle d'utilisation de ce dernier.

Puis sur la base d'une régression linéairelogarithmique de la consommation de travail par $\$ 1,000.00$ de valeur ajoutée aux taux de salaires, c'est la dérivation d'estimations de l'élasticité entre le capital et le travail pour vingt-quatre catégories bien définies d'industries monufacturières qui est l'objet de considérations particulières. Les implications de la variabilité de cette substitution sur les avantages comparés eu égard au commerce international sont examinées dans une outre partie de cet exposé.

Dans ces premiers chapitres le toux de rendement du copital joue un rôle d'arrièreplan, voilà pourquoi dans les deux suivants s'opplique-t-on à l'évaluation des différences dans le prix de ce facteur de production pour quelques régions pour lesquelles des données comporables peuvent être obtenues. En examinant le contenu du chapitre six, on remarque que ces estimés de rendement du capitol sont utilisés pour différentes fins comme vérifier l'hypothèse d'une tendance à des rendements égaux dans différents commerces, constater l'efficacité globale du copital industriel entre les pays paurres et les pays riches, et découvrir les différences dans la profitabilité relative de différentes industries dans différents pays.

Enfin, un résumé des principales découvertes de l'étude nous offrant quelques réflexions sur les implications politiques de ces découvertes et certaines suggestions pour des recherches antérieures viennent terminer les propos de l'auteur.

Ronald Pleau

\section{Les installations électriques en moyenne} tension, J. Boulouvard, Entreprise moderne d'édition, Paris, 1964, 78 pages.

Dans son avant-propos, l'auteur avertit le lecteur que son étude a pour but de renseigner les chefs d'étoblissements industriels, militaires et médicaux qui comportent plusieurs bâtiments espocés les uns des autres dans lesquels une instollation électrique est à réaliser en première exécution ou en remplacement d'une ancienne.

L'auteur propose donc d'étudier les installations de moyenne tension (jusqu'd 20,000 volts), qui permettent une distribution d'énergie plus puissante et plus étendue.

Après avoir roppelé l'utilité et l'applicotion de la moyenne tension et les différentes coractéristiques à définir pour son implantotion, l'ouvrage étudie en détail chacune des parties de l'installation à savoir le poste de livraison, le poste de transformation, les côbles, le matériel de comptage, les circuits de terre, les verrouillages et éclairage des locoux.

Suivent ensuite toutes les informations nécessaires à l'installation et d̀ l'exploitotion de ces systèmes telles l'étude et la prévision des incidents électriques dans l'instal'lation, nombre et noture des cellules moyenne-terision, choix du fabricant et des installateurs, implantation des bâtiments, mise en service, entretien, vérification et tarification de ces installations.

Cet ouvrage de défrichement, si on peut dire, puisquaucune documentation complète jusqu'à ce jour n'existoit sur ce sujet, est remarquable par so concision et sa précision. II ne peut qu'être des plus utiles à tous les chefs d'exploitation, les techniciens, les ingénieurs électriciens et les architectes comme ouvrage de bose afin d'ovoir une idée générale concernant ces nouveoux produits le la science.

\section{Ronald Pleau}

Les opprentis scolarisés, par Robert Schiélé et André Moujardet, dans la Collection - L'Enquête et l'Action *, les Editions ouvrières, Paris, 328 pages.

Peu d'enquêtes présentent outant d'intérêt que celle qui nous est préseritée dons cet ouvroge.

A l'origine, quelques apprentis en école veulent sovoir ce que pensent leurs camarades apportenant d̀ d'autres étoblissements. 
Et voilà qu'ils déclenchent une étude systémotique à laquelle seront mêlés plus de cina mille garçons de 14 d̀ 17 ans, préparont le C.A.P. ou le B.E.I., répartis sur toute la France, à peu près la moitié dans des écoles confessionnelles. Le seul exposé de lo méthode est déjà possionnant: les jeunes ne se sont pas contentés de répondre honnêtement ò un questionnaire très étoffé, ils $y$ ont joint en annexe leurs réflexions et commentaires qui sont parfois savoureux, ainsi d'ailleurs que les interviews. Quant aux résultats, ils sont extrêment intéressonts, car c'est la première fois qu'une enquête aussi approfondie est faite sur un milieu aussi déterminé.

Le sérieux des réponses est froppant: moins de quatre pour cent ont été reconnues non volables. Sont onalysés: le cheminement, la maturation, la culture et l'adap- tation professionnels, l'attitude vis-ò-vis du trovail, de l'argent, des valeurs, la vie morale et religieuse, la camaraderie et les loisirs. Enfin, dans un dernier chapitre, les apprentis apprécient leurs chances et se comparent oux élèves des lycées.

Cette étude de mentalité intéressero ou premier chef les psychologues, les orienteurs, les élucateurs, les parents, tous ceux qui, pour comprendre la jeunesse, ne se contentent pas de sentiment. Elle sera aussi profitable à tous ceux qui s'occupent du problème de l'emploi, de l'évolution économique, des loisirs.

Signalons, enfin, les très nombreuses références bibliogrophiques, les onnexes et la table récopitulative qui font de ce travail une véritable petite somme sur la mentalité de l'apprenti en école.

\section{PUBLICATIONS RECENTES RECENT PUBLICATIONS}

\section{GÉNÉRALITÉS}

\&hy Automation? $\$$, Tom Rose, The Freeman, Vol. 15, No. 7, July 1965, pp. 3-11.

\&Le progrès technique et ses conséquences socioles dans l'industrie textile française $\gg$, Revue internationale du Trovail, Vol. 92, no 1, juillet 1965, pp. 55-67.

- Training Foreign Notionals for Employment with U.S. Componies in Developing Countries $\gg$, Manpower Research, U.S. Department of Labor, Bulletin number 7, February 1965, pp. $1-14$.

Recherches Sociogrophiques, vol. VI, no I, jonvier-avril 1965, numéro sur Les Classes sociales au Conada français, pp. 9-87.

Jobs, Wages, and Changing Technology: Recent Experience, by Robert $L$. Aronson, New York State School of Industrial and Labor Relations, Cornell University, Ithaca, New York, Bulletin 55, July 1965, pp. 1-74

L'Apartheid au Pilori, Le dossier de lo Fédération syndicale mondiale, pp. 1-34.
« Conférence en Amérique du Nord sur les tâches automatisées », La Gazette du Travail, Vol.. LXV, no 2, février 1965, pp. 110117.

«La propriété et l'entreprise \$, par J.-P. Latteur, Bulletin Social des Industriels, no 317, mai 1965, pp. 276-281.

«Social Issues and Poverty Research: A Commentary $\gg$, by Berton $H$. Kaplan, The Journal of Social Issues, Vol. XXI, No. I, January 1965, pp. 1-10.

\&éclin, renouveau ou métamorphose du capitalisme $\gg$, by L. Bourcier de Carbon, Droit Social, no 5, mai 1965, pp. 277-287.

«Le progrès technique et la planification sociale dans l'entreprise », par Gunter Friedrichs, Revue internationale du travail, vol. 92, no 2, ooût 1965, pp. 97-113.

《Les grandes manoeuvres du patronat ${ }_{\text {, }}$ por P. Belmont, Economie $\&$ Humanisme, 162 juillet, oout 1965, pp. 36-45. 